\title{
Solo-Brita em Bases de Pavimentos Flexíveis: Avaliação quando à Fadiga Utilizando o MeDiNa
}

\author{
Soil-Gravel in Flexible Pavement Bases: Evaluation of Performance in Fatigue Using the MeDiNa
}

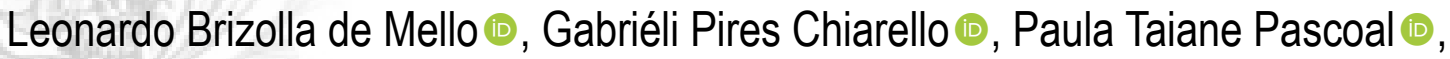 \\ Magnos Baroni $\odot$, Luciano Pivotto Specht
}

Universidade Federal de Santa Maria, Programa de Pós-Gradução em Engenharia Civil, Santa Maria, RS, Brasil

E-mails: leobrmello@hotmail.com; gabrieli.chiarello@gmail.com; ptpascoal@hotmail.com; magnos.baroni@ufsm.br; luspecht@ufsm.br

\section{Resumo}

A aplicação de materiais alternativos em substituição a bases e sub-bases de pavimentos compostas por materiais comumente aplicados em obras rodoviárias pode ser uma excelente alternativa, visando economia e um desempenho satisfatório a novos trechos. Sabe-se que o método oficial vigente de dimensionamento se priva de importantes aspectos correlatos a parâmetros de capacidade portante dos materiais, por isso tornou-se necessário o desenvolvimento de uma nova metodologia de dimensionamento empírico-mecanicista, o MeDiNa. Um dos fatores considerados por essa nova metodologia é o comportamento resiliente dos materiais. Assim, neste trabalho, foram analisadas amostras de solo compactado na energia modificada do ensaio Proctor e propostas três misturas de solo argiloso laterítico e brita descontínua, em porcentagens de 25, 30 e 35\% em massa de solo, para aplicação em camadas estruturais de pavimentos, de forma a compreender qual delas representaria o melhor comportamento quando aplicados em rodovias de baixo, médio e volume de tráfego real, além de analisar a viabilidade econômica. Os resultados demonstram bons comportamentos resilientes das amostras, com destaque à mistura 2 (intermediária) que teve coeficientes de correlação da ordem de 0,9 . Foi simulada a aplicação dessa mistura como camada de base de uma estrutura de um pavimento já existente (pavimento referência) e analisado o seu comportamento quanto à fadiga, resultando em um desempenho superior, com menor percentual de área trincada ao longo da vida útil e redução de custos de até $60 \%$.

Palavras-chave: Dimensionamento Empírico-mecanicista; Módulo de Resiliência; Fadiga

\begin{abstract}
The application of alternative materials in replacement of bases and sub-bases composed by materials that are commonly used on road construction can be an excellent alternative, aiming at savings and satisfactory performance to the new highways. It is known that the current design method deprives itself of important aspects related to parameters of the resistant capacity of the materials, therefore it became necessary to develop a new methodology of empirical-mechanistic design, called MeDiNa. One of the factors considered by this new methodology is the resilient behavior of the materials. Thus, in this work, soil samples compacted in intermediate energy by Proctor test method were analyzed and three mixtures of lateritic clay soil and batch gravel were proposed, in percentages of 25,30 and $35 \%$ of soil, to be used in road structural layers, in order to understand which one would represent the best behavior when applied to the low, medium and high volume of traffic, in addition to an economic viability analyze. The results demonstrate good resilient behavior of the samples, with emphasis on mixture 2 (intermediate mixture), which had correlation coefficients in order of 0.9 . The application of this mixture was simulated as the base layer of an existing pavement structure (reference pavement) and its fatigue behavior was analyzed, resulting in a superior performance, with lower percentage of cracked area over its useful life, and a cost reduction up to $60 \%$.
\end{abstract}

Keywords: Empirical-mechanistic Design; Resilient Modulus; Fatigue 


\section{Introdução}

A aplicação de materiais e misturas alternativas em camadas de base e sub-base de pavimentos, como composições de solo e brita, podem ser um caminho financeiramente viável e ambientalmente adequado (Villibor et al., 2009). Para tal, é substancial, em um dimensionamento empírico-mecanicista, compreender a deformabilidade dos materiais que compõem a estrutura do pavimento (Soares et al., 2009; Bernucci et al., 2010; Barros et al., 2019). Outro fator preponderante é compreender como as relações tensão-deformação de cada material (Prado et al., 2020), advindas de ensaios de módulo de resiliência (MR) e deformação permanente (DP), influenciam no comportamento das misturas dependentes dos estados de tensões aplicados em ambos os ensaios. Estas propriedades foram consideradas na concepção de um novo método de dimensionamento de pavimentos flexíveis no Brasil, denominado Método de Dimensionamento Nacional de Pavimentos (MeDiNa).

Dessa forma, torna-se imprescindível caracterizar os materiais granulares e terrosos, que compõem as camadas adjacentes ao revestimento asfáltico, por meio de atributos obtidos através de ensaios realizados no equipamento triaxial de cargas repetidas, capaz de reproduzir as condições de campo, simulando a passagem do veículo em um ponto na superfície do pavimento e verificar o efeito produzido nas camadas da estrutura (Medina \& Motta, 2015; Norback \& Motta, 2018; Franco \& Motta, 2018; Lima et al., 2018, 2019).

Neste sentido, apresentar-se-á a caracterização física e química dos materiais abordados, o comportamento resiliente do solo natural e de três misturas de latossolo argiloso e material britado, em diferentes porcentagens, todos compactados na energia modificada do ensaio Proctor, determinado a partir do equipamento triaxial de cargas repetidas. A fim de demonstrar a aplicação de solo-brita, a mistura com granulometria de melhor desempenho foi considerada como camada de base alternativa de um pavimento referência, já implantado no noroeste do Rio Grande do Sul, os quais foram dimensionados através de conceitos mecanicistas pelo MeDiNa, avaliados segundo seu desempenho técnico e comparados em termos de custo por meio do Sistema de Custos Referenciais de Obras (SICRO) do DNIT e da composição de preços do Departamento Autônomo de Estradas de Rodagem (DAER, 2019).

\section{Materiais e Métodos}

A pesquisa foi realizada em quatro etapas: (i) coleta de amostras de brita e amostras deformadas de solo em suas respectivas jazidas, (ii) ensaios laboratoriais, (iii) análises de estruturas propostas por meio do software MeDiNa v. 1.1.3 de setembro/2019 e (iv) avaliação de sua viabilidade econômica. Em vista de que a distribuição granulométrica é um dos aspectos mais importantes em relação ao comportamento de misturas solo agregado (Yoder \& Witczak, 1975; Esfahani, 2018), os estudos se baseiam em ensaios laboratoriais de corpos de prova de solo laterítico e misturas de solo e brita descontínua (SB) em três composições diferentes que embasarão às análises quanto à fadiga, conforme apresentado na Tabela 1 .

\subsection{Materiais}

O solo, Figura 1 (A), foi coletado em uma jazida no município de Cruz Alta, região noroeste do estado do Rio Grande do Sul, localizado nas coordenadas 28 37'39.20" sul e $53^{\circ} 37^{\prime} 31.50$ " oeste. Coletou-se material do horizonte pedogenético B do talude apresentado na Figura 1 (B). A escolha desse ocorreu devido ao emprego efetivo em obras rodoviárias, em conta da menor atividade biológica e, consequentemente, menor ocorrência de matéria orgânica, como se observa no horizonte $\mathrm{A}$, e a inexistência de sedimentos oriundos da rocha mãe, como no horizonte $\mathrm{C}$, informações obtidas após a caracterização em laboratório dos três horizontes.

$\mathrm{O}$ agregado pétreo é oriundo da Pedreira São Juvenal, Figura 2 (A), localizada na cidade de Cruz Alta sob as coordenadas $28^{\circ} 37^{\prime} 30.99^{\prime \prime}$ sul e 53⒊' $10.80^{\prime \prime}$ oeste. Ele foi coletado das pilhas da pedreira, Figura 2 (B), e é formado por rocha ígnea extrusiva, de cor cinza escuro e tonalidades parciais de verde e vermelho (Back, 2018). Neste estudo, foi utilizada uma composição descontínua de britas 3/4" (passante na peneira de $19,5 \mathrm{~mm}$ e retido na peneira de $9,5 \mathrm{~mm}$ ) e britas $3 / 8$ " (passante na peneira de $9,5 \mathrm{~mm}$ e retido na peneira de $4,8 \mathrm{~mm}$ ).

\begin{tabular}{lcccc}
\hline Material & Solo Laterítico & Brita 3/4” & Brita 3/8” & Energia de compactação \\
\hline Solo natural & $100 \%$ & - & - & Proctor modificado \\
SB 1 & $25 \%$ & $45 \%$ & $30 \%$ & Proctor modificado \\
SB 2 & $30 \%$ & $40 \%$ & $30 \%$ & Proctor modificado \\
SB 3 & $35 \%$ & $35 \%$ & $30 \%$ & Proctor modificado \\
\hline
\end{tabular}

Tabela 1 Composição dos materiais propostos para o estudo. 


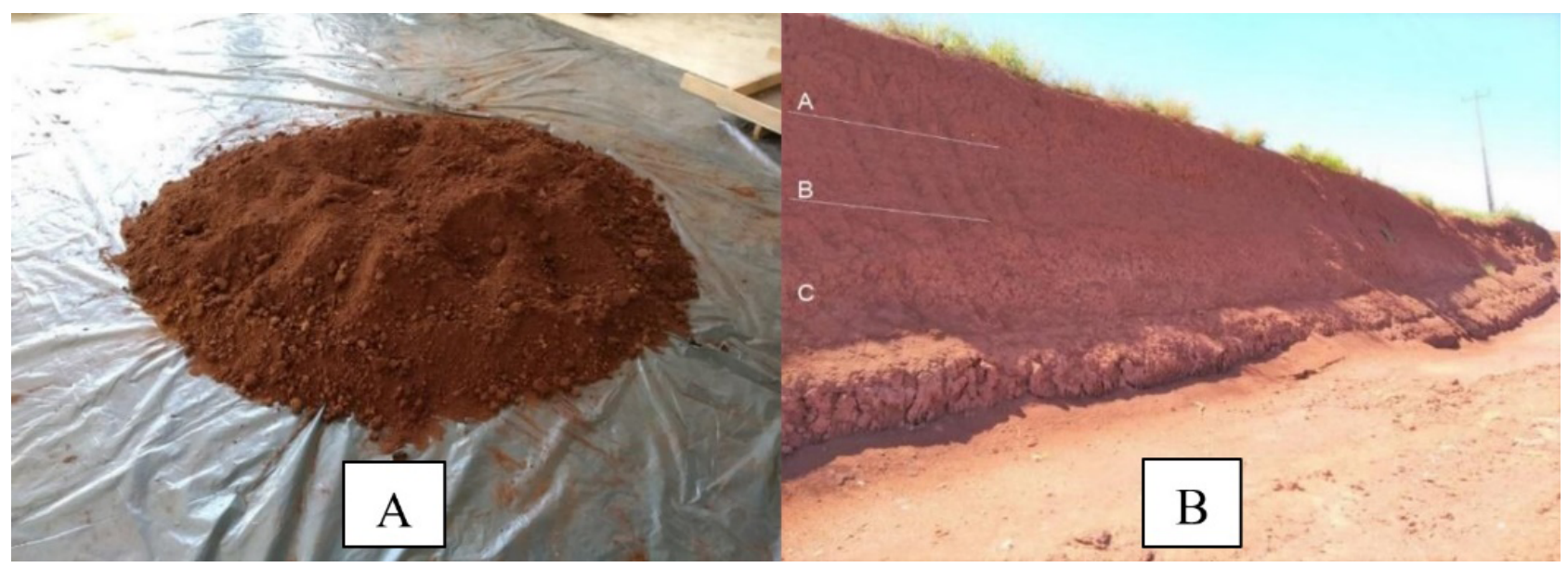

Figura 1 A. Solo coletado; B. Talude natural da jazida.

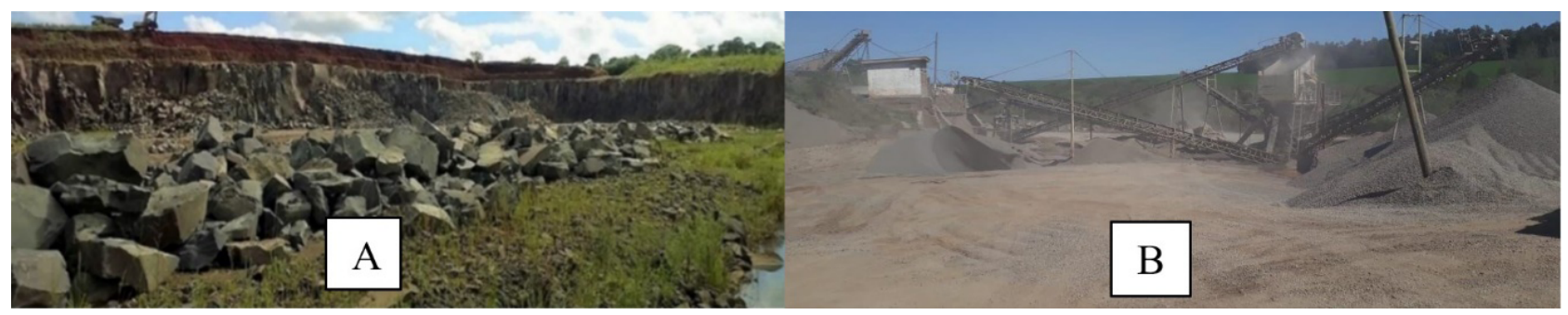

Figura 2 A. Jazida de coleta das britas; B. Pilhas com diferentes frações granulométricas.

\subsection{Caracterização Física e Química}

O solo foi caracterizado fisicamente com base nos resultados dos limites de Atterberg (ABNT, 2016a, 2016c), ensaio de granulometria por peneiramento, sedimentação (ABNT, 2016d), massa específica real (ABNT, 2016b), classificados pelas metodologias tradicionais (Sistema Unificado de Classificação de Solos - SUCS e Transportation Research Board da American Association of State Highway and Transportation Official - TRB/AASHTO) e pela metodologia MCT (DNER, 1996). Quimicamente, o solo foi caracterizado por meio da Fluorescência de Raio-X por Energia Dispersiva (FRX ED), com equipamento S2 Ranger Bruker.

A brita foi caracterizada por Back (2018) em ensaios de granulometria por peneiramento (DNER, 1998b), massa específica real (DNER, 97b), absorção dos agregados graúdos (ABNT, 2011), índice de lamelaridade (DAER, 2001), equivalente de areia (DNER, 1997a) e Abrasão Los Angeles (DNER, 1998a).

\subsection{Caracterização Mecânica}

As curvas de compactação do solo e de cada mistura seguiram os preceitos da Rede 04 (Petrobras, 2010), que determina o uso de cilindro tripartido de $10 \mathrm{~cm}$ de diâmetro e $20 \mathrm{~cm}$ de altura, para determinar a umidade ótima e o peso específico aparente máximo dos materiais. Foram realizadas quatro curvas de compactação na energia modificada, uma para cada mistura e uma para o solo, em compactador mecânico, com soquete de $2500 \mathrm{~g}$ de massa, na altura de queda de $30,5 \mathrm{~cm}$, com dez camadas de 56 golpes cada.

Em posse da umidade ótima e densidade máxima, para determinar as propriedades elásticas das misturas de solo e solo-brita, moldaram-se corpos de prova (CP) em triplicata. Em seguida, os CPs foram submetidos a ensaios de módulo de resiliência no equipamento triaxial dinâmico conforme a normativa DNIT (2018).

Com os módulos resilientes de cada amostra, há alguns modelos que, a fim de se verificar o comportamento resiliente, concatenam aos esforços aplicados e às constantes estabelecidas laboratorialmente. Nesse sentido, através do software Statistica v. 10, utilizou-se modelos matemáticos, consagrados pela literatura, de comportamento elástico não linear, considerando a relação entre o Módulo de Resiliência e às tensões impostas, obtidas por meio de regressão, para a obtenção dos parâmetros, sendo eles: modelo de Biarez (1962), modelo de Svenson (1980), modelo composto (Pezo et al., 1992), modelo Universal (AASHTO, 2004) e modelo das Tensões Principais (Medina \& Motta, 2015). 
A Tabela 2 apresenta as equações à nomenclatura adotada para cada modelo (Seed et al., 1967; Medina \& Preussler, 1980; Svenson, 1980; Motta, 1991; Li \& Selig, 1994; Werk, 2000; Guimarães et al., 2001; AASHTO, 2004; Medina \& Motta, 2015).

O critério considerado para avaliar qual modelo representa melhor o comportamento resiliente do material foi o coeficiente de determinação $\left(\mathrm{R}^{2}\right)$, também denominado coeficiente de correlação.

\subsection{Análise Mecanicista e Econômica}

A análise mecanicista ocorreu através do software MeDiNa v. 1.1.3.0 e foi realizada para compreender o comportamento quanto à fadiga, relacionado ao módulo resiliente, cujo limite de dano máximo aceito pelo software é de $30 \%$ de área trincada até o fim da vida útil, para o sistema arterial principal, considerado nos dimensionamentos. Foram avaliadas duas estruturas de pavimentos considerando o tempo de projeto de 10 anos, sob três volumes de tráfegos: um baixo $\left(\mathrm{N}: 1,0 \times 10^{6}\right)$, outro médio $\left(\mathrm{N}: 5,0 \times 10^{6}\right)$, conforme recomendado por Ceratti et al. (2015), e um último ligado ao volume de projeto utilizado para a construção do acesso na ERS $342\left(\mathrm{~N}: 3,57 \times 10^{7}\right)$, ou seja, três análises para cada estrutura.

A primeira estrutura, abordada nesta pesquisa como pavimento referência, é composta por duas camadas de concreto asfáltico, base de brita graduada simples, sub-base granular e subleito de solo laterítico argiloso. Essa estrutura simula o pavimento existente em termos de materiais e espessuras, projetado e executado no trevo de acesso Norte a cidade de Cruz Alta/RS. A segunda estrutura diz respeito a um pavimento alternativo, em que a base e sub-base foram substituídas por uma única camada formada pela mistura de melhor comportamento resiliente, posteriormente analisada às condições de tráfego propostas. A Figura 3 apresenta a disposição das camadas bem como os materiais considerados em cada estrutura.

O subleito avaliado em ambas estruturas faz referência aos resultados das amostras ensaiadas na energia modificada com resultados apresentados no decorrer deste trabalho. Já o revestimento asfáltico considerado na camada inferior é resultado da mistura asfáltica avaliada por Rossato (2015). Os materiais que compõem a camada de base e sub-base, além da segunda camada de revestimento asfáltico, são componentes do banco de dados do MeDiNa, com propriedades apresentadas na Tabela 3.Em posse das estruturas de pavimento dimensionadas e avaliadas no MeDiNa, foram analisados os custos inerentes à implantação de $1 \mathrm{~km}$ através da Composição de Preços de maio de 2019 do DAER/RS e $1 \mathrm{~km}$ através do SICRO do DNIT (2019), de outubro de 2019 e em posse de dados da Agência Nacional do Petróleo, Gás Natural e Biocombustíveis (ANP, 2020) referentes a precificação média do asfalto em fevereiro de 2020. Compõem o primeiro orçamento somente a análise da diferença de custos da execução das camadas de base e sub-base de cada uma das estruturas, sem considerar as demais porções do pavimento. Já o levantamento de custos do DNIT, faz menção aos custos diretos da implantação, como as movimentações de terra, execução das camadas de base, pinturas de ligação e imprimação das camadas e sistema de sinalizações do trecho.

\begin{tabular}{lc}
\hline Modelos & Equação \\
\hline$k \sigma_{3}$ - Biarez (1962) - tensão confinante & $M_{R}=k_{1} \cdot \sigma_{3}^{k_{2}}$ \\
$k \sigma_{d}$ - Svenson (1980) - tensão desvio & $M_{R}=k_{1} \cdot \sigma_{d}^{k_{2}}$ \\
Composto - Pezo et al. (1992) & $M_{R}=k_{1} \cdot \sigma_{3}^{k_{2}} \cdot \sigma_{d}^{k_{3}}$ \\
Universal - AASHTO (2004) & $M_{R}=k_{1} \cdot \rho_{a}\left(\frac{\theta}{\rho a}\right)^{k_{2}} \cdot\left(\frac{\tau_{o c t}}{\rho a}+1\right)^{k_{3}}$ \\
$k \theta$ - Invariante de tensões - tensões principais & $M_{R}=k_{1} \cdot \theta^{k_{2}}$
\end{tabular}

Onde: $M_{R}$ : módulo de resiliência; $\sigma_{3}$ : tensão confinante; $\sigma_{d}$ : tensão desvio; $\theta$ : tensão principal; $\tau_{\text {oct }}$ : tensão octatédrica; $\rho_{a}:$ pressão atmosférica; $k_{1}$, $k_{2}$ e $k_{3}$ : parâmetros de resiliência determinados experimentalmente.

Tabela 2 Modelos matemáticos utilizados. 

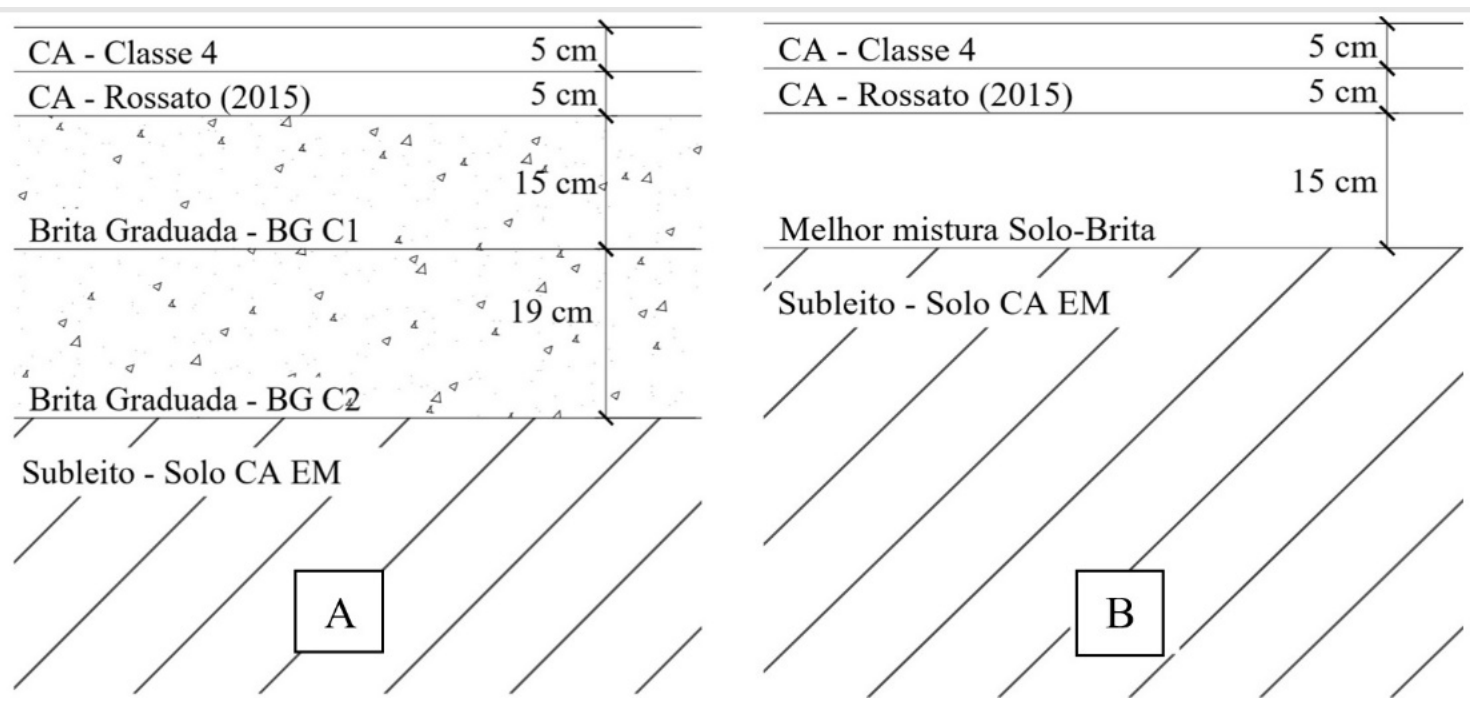

Figura 3 A. Estrutura referência; B. Estrutura alternativa.

\begin{tabular}{lcccc}
\hline Parâmetros & Alto Módulo - EVA & $\begin{array}{c}\text { Concreto Asfáltico CA } \\
\text { Rossato }\end{array}$ & $\begin{array}{c}\text { Brita Graduada } \\
\text { Gnaisse C1 }\end{array}$ & $\begin{array}{c}\text { Brita Graduada } \\
\text { Gnaisse C2 }\end{array}$ \\
\hline Nomenclatura utilizada & EVA & Rossato $(2015)$ & BG C1 & BG C2 \\
Coeficiente de Poisson & 0,30 & 0,30 & 0,35 & 0,35 \\
Contato & Aderido & Não aderido & Não aderido & Não aderido \\
M. Especifica $\left(\mathrm{g} / \mathrm{cm}^{3}\right)$ & 2,42 & 2,21 & 2,29 & 2,27 \\
Abrasão LA $(\%)$ & 18,70 & 10,82 & - & - \\
Energia de Compactação & - & - & Modificada & Modificada \\
MR linear $(\mathrm{MPa})$ & 10982 & 4901 & 279 & 259 \\
Ensaio Fadiga $-\mathrm{k}_{1}$ & $1 \mathrm{e}-27$ & $4 \mathrm{e}-5$ & - & - \\
Ensaio Fadiga - $\mathrm{k}_{2}$ & $-7,38$ & $-1,99$ & - & - \\
\hline
\end{tabular}

Tabela 3 Propriedades dos materiais adotados.

\section{Resultados e Discussões}

\subsection{Caracterização Física e Química}

A Figura 4 apresenta a análise granulométrica do agregado, em diferentes amostragens, e dos solos, com e sem o uso do hexametafosfato de sódio. Esse permite a melhor distribuição das partículas ao desaglomerar os grumos de solos finos, à medida que, sem o uso do agente químico, a curva traduz um material de granulometria mais contínua e de maiores dimensões. Em um conjunto geral, percebe-se pouca variação dos resultados dos ensaios realizados em triplicatas, apresentados na Tabela 4 , o que comprova a qualidade dos resultados. O solo é composto por aproximadamente $41 \%$ de argila, $26 \%$ de silte e $33 \%$ de areia (fina e média).
De acordo com a SUCS, o material é um silte de alta plasticidade (MH). Já a TRB/AASHTO, apresenta o solo com um comportamento considerado de sofrível a mau ao se empregar em subleitos rodoviários, por se enquadrar no subgrupo A-7-6 com índice de grupo (IG) igual a 11, isto é, materiais de elevada variação volumétrica.

Conforme a classificação MCT, o solo foi categorizado como um latossolo argiloso (LG'), com índice c'variando de 2,27 a 2,44 e índice e' entre 0,67 e 0,71. Com a análise da variabilidade de desprendimento das amostras, na classificação MCT-M (Vertematti, 1988), verificou-se que a classificação dos dois exemplares do solo mantém-se constantes, ou seja, classificam-se ainda com LG'. Solos lateríticos possuem a presença de hidróxidos de alumínio e ferro, compostos que participam do processo natural de cimentação do elemento. Os resultados da composição química destacam a presença de dióxido de silício e óxidos de ferro e alumínio em significativas porcentagens. 


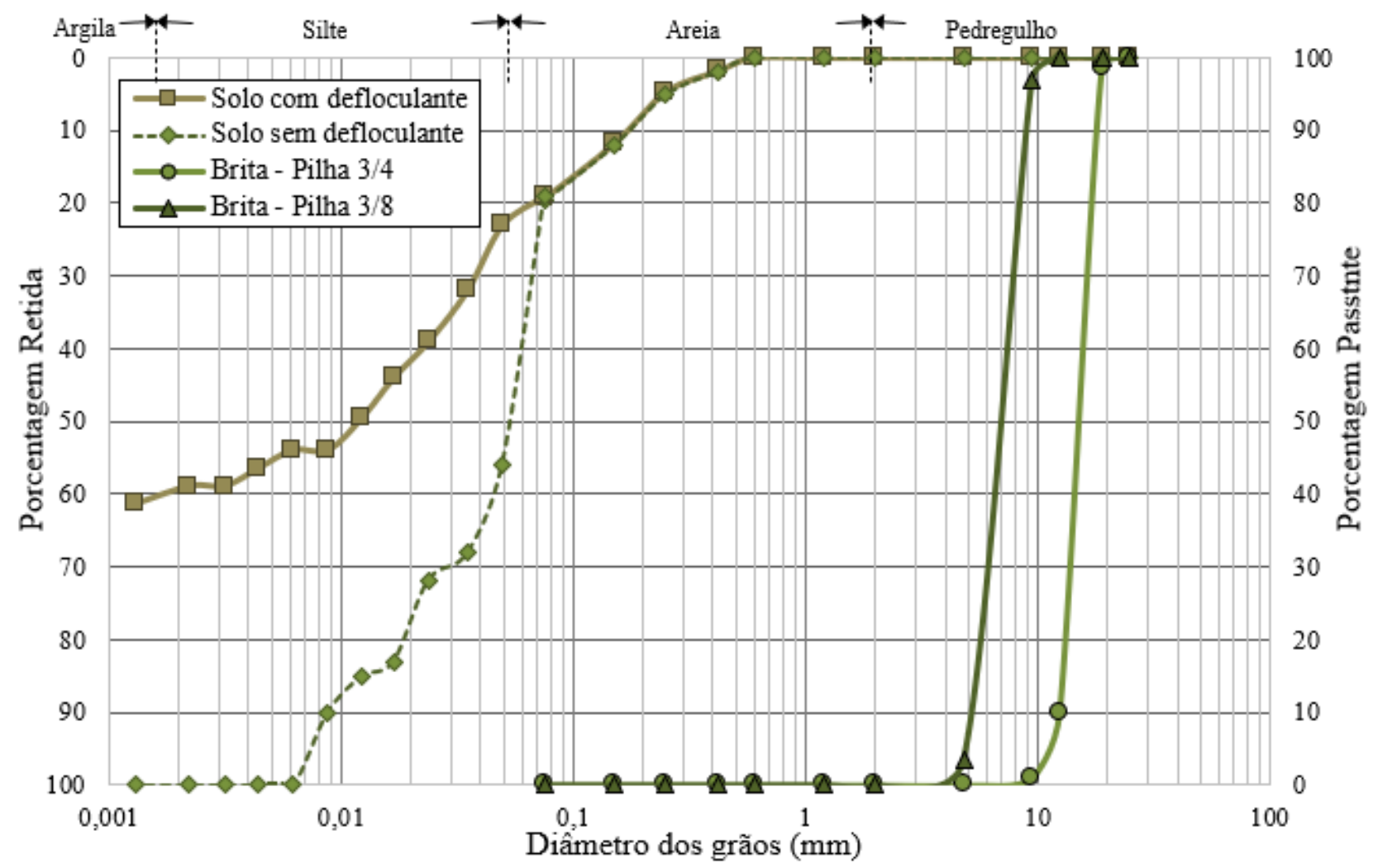

Figura 4 Curvas granulométricas do solo e britas.

Os resultados das análises físicas, obtidos por Back (2018), estão na Tabela 4. Nota-se que, em termos de absorção, massa específica real e porosidade aparente, as britas de menores dimensões apresentam índices superiores, enquanto que nos demais aspectos as britas $3 / 4$ " possuem valores superiores.

\subsection{Caracterização Mecânica}

Para se tornar possível realizar a análise do comportamento resiliente do solo e das amostras SB, fez-se necessário realizar o ensaio de compactação, a fim de obter os parâmetros de umidade ótima e massa específica aparente seca. A Figura 5 apresenta as curvas de compactação das misturas de solo e SB na energia modificada.

Os módulos resilientes foram ponderados através do equipamento triaxial de cargas repetidas em um total de oito corpos de prova. Três deles considerando a mistura SB 1, dois corpos de prova para a SB 2 e três as amostras de SB 3. Salienta-se que, conforme a normativa do DNIT
(2018), ao realizar a moldagem dos corpos de prova, estes serão considerados válidos se apresentarem uma variação de umidade de até $1 \%$ da estipulada no ensaio de compactação. A Tabela 5 apresenta os resultados da moldagem dos corpos de prova.

Em posse dos resultados, foi possível determinar os parâmetros resilientes a partir de avaliações no software Statistica para o conjunto de amostras de cada condição ensaiada. Assim, ao avaliar o conjunto de amostras (I+II+III), foi considerada uma quantidade maior de dados, visando eliminar a possibilidade de erros em relação à rigidez do material. Foram estudados cinco modelos estatísticos para obter o comportamento resiliente, a fim de verificar quais dos modelos se adequam melhor ao comportamento do solo natural e das misturas SB. Os resultados estão apresentados na Tabela 6.

A Figura $6(\mathrm{~A}, \mathrm{~B}, \mathrm{C}, \mathrm{D})$ apresenta os gráficos do modelo com os melhores coeficientes de correlação, o Composto, que relaciona a tensão confinante e desvio à obtenção dos MR's. 


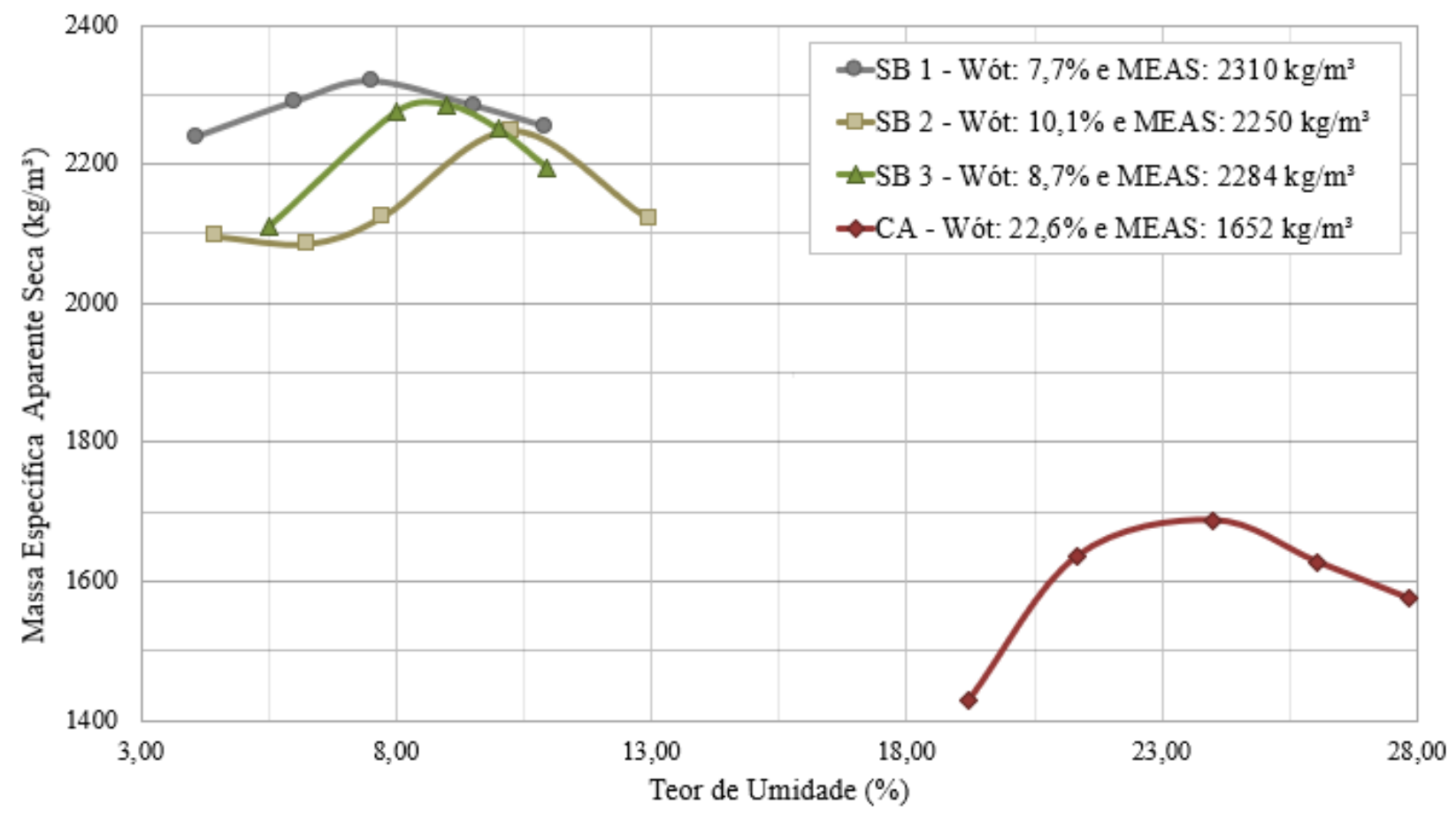

Figura 5 Curvas de compactação na energia modificada e cilindro tripartido

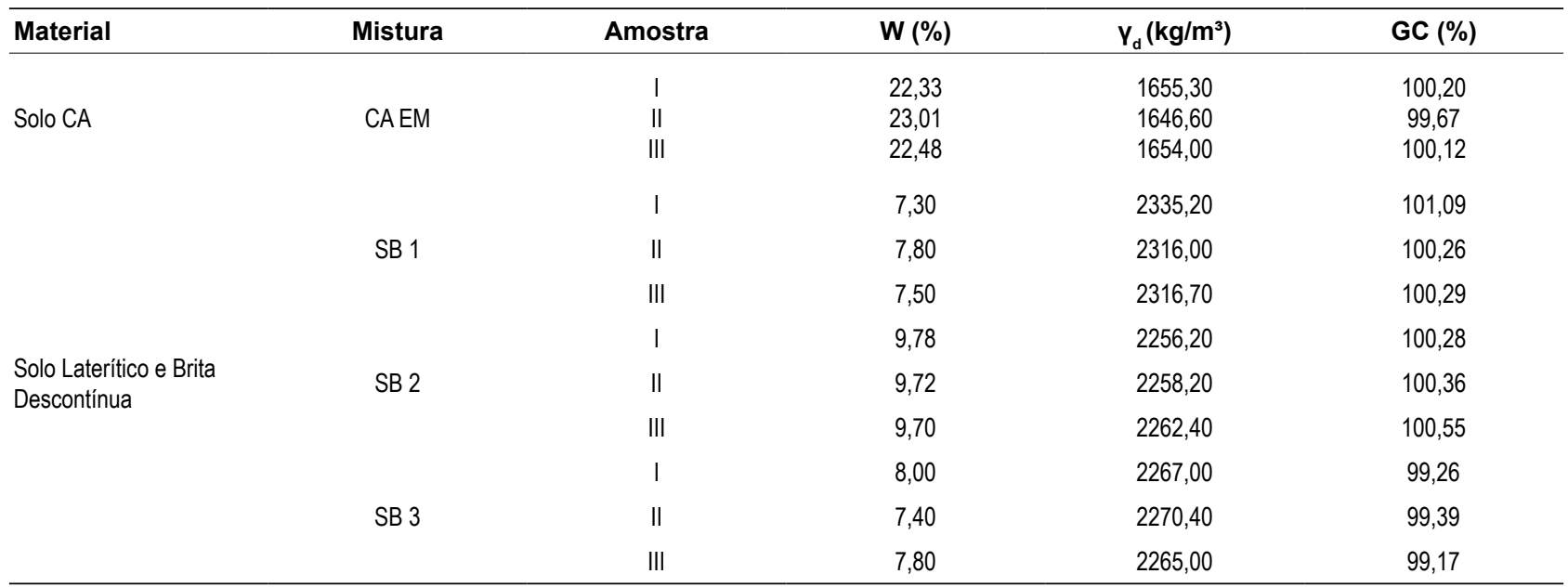

*Amostra invalidada em virtude de erro na montagem do equipamento triaxial.

Tabela 5 Propriedades dos corpos de prova moldados.

Nota-se que o modelo Svenson não apresentou boas correlações para nenhum dos materiais propostos, desta forma, seus resultados não serão considerados nas análises a seguir. Ainda que o modelo Universal tenha apresentado correlações satisfatórias, o modelo Invariante de Tensões, Biarez e Composto apresentaram $\mathrm{R}^{2}$ superiores, os quais são apresentados na Figura 7 com um resumo dos valores de MR médio de cada material.
O modelo Composto apresentou as melhores correlações, com destaque ao solo natural e à mistura SB 2, cujos módulos de resiliência médios foram de $351 \mathrm{MPa}$ e $356 \mathrm{MPa}$ e $\mathrm{R}^{2}$ de 0,93 e 0,92 , respectivamente. $\mathrm{O}$ valor positivo do parâmetro $\mathrm{k} 2$ indica que com o incremento da tensão confinante, há um aumento no módulo de resiliência. $\mathrm{O}$ fato de o parâmetro $\mathrm{k} 3$, vinculado a tensão desvio nesse modelo, apresentar um valor negativo para as misturas de 


\begin{tabular}{|c|c|c|c|c|c|c|}
\hline Modelo & Amostra & k1 & k2 & k3 & $\mathbf{R}^{2}$ & MR (MPa) \\
\hline \multirow{4}{*}{ Biarez $\left(\sigma_{3}\right)$} & CA EM - $|+||+|||$ & 1724,19 & 0,50 & - & 0,91 & 352 \\
\hline & SB 1 - |+||+|II & 1590,65 & 0,63 & - & 0,94 & 285 \\
\hline & SB $2-|+||+|||$ & 1383,45 & 0,49 & - & 0,86 & 357 \\
\hline & SB $3-1+||+|| \mid$ & 1099,27 & 0,48 & - & 0,63 & 293 \\
\hline \multirow{4}{*}{ Invariante de tensões $(\theta)$} & CA EM - $|+||+|||$ & 721,03 & 0,46 & - & 0,89 & 349 \\
\hline & SB 1 - |+|I+|II & 541,64 & 0,58 & - & 0,87 & 281 \\
\hline & SB $2-|+\|+|\||$ & 755,86 & 0,55 & - & 0,64 & 404 \\
\hline & SB $3-|+||+|||$ & 472,40 & 0,41 & - & 0,52 & 292 \\
\hline \multirow{4}{*}{ Svenson $\left(\sigma_{d}\right)$} & CA EM - $|+||+|||$ & 729,83 & 0,28 & - & 0,62 & 355 \\
\hline & SB $1-|+||+|||$ & 657,88 & 0,40 & - & 0,61 & 280 \\
\hline & SB $2-|+||+|||$ & 624,33 & 0,25 & - & 0,39 & 358 \\
\hline & SB $3-1+\|+|| \mid$ & 512,60 & 0,26 & - & 0,30 & 291 \\
\hline \multirow{4}{*}{ Composto } & CA EM - I+ II+|II & 1640,16 & 0,42 & 0,08 & 0,93 & 351 \\
\hline & SB 1 - I+||+|II & 1593,00 & 0,64 & $-0,02$ & 0,94 & 267 \\
\hline & SB $2-|+||+|||$ & 1542,54 & 0,66 & $-0,16$ & 0,92 & 356 \\
\hline & SB $3-|+||+|||$ & 1184,18 & 0,61 & $-0,13$ & 0,66 & 248 \\
\hline \multirow{4}{*}{ Universal } & CA EM - I+ II+III & 1185,26 & 0,65 & $-0,55$ & 0,94 & 351 \\
\hline & SB $1-|+||+|||$ & 409,54 & 0,43 & 3,18 & 0,70 & 318 \\
\hline & SB $2-|+||+|||$ & 698,30 & 0,52 & 0,57 & 0,65 & 359 \\
\hline & SB $3-1+\|+|| \mid$ & 439,57 & 0,35 & 1,69 & 0,42 & 329 \\
\hline
\end{tabular}

Tabela 6 Parâmetros resilientes para o conjunto de amostras de acordo com os modelos.

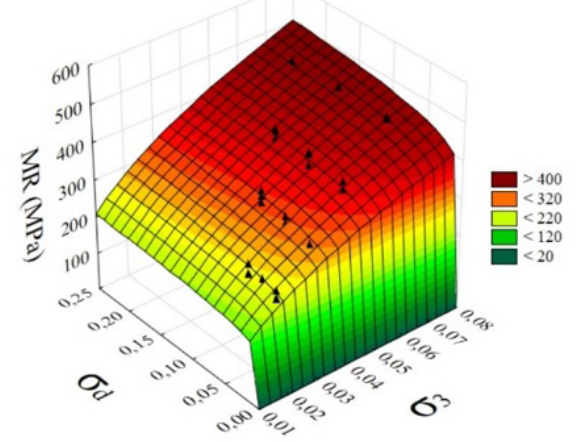

A

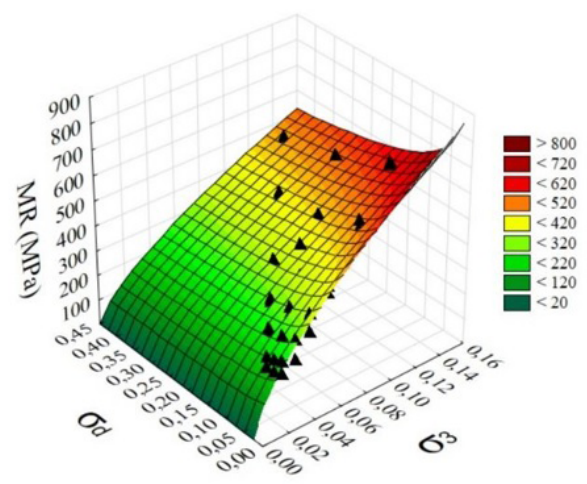

C

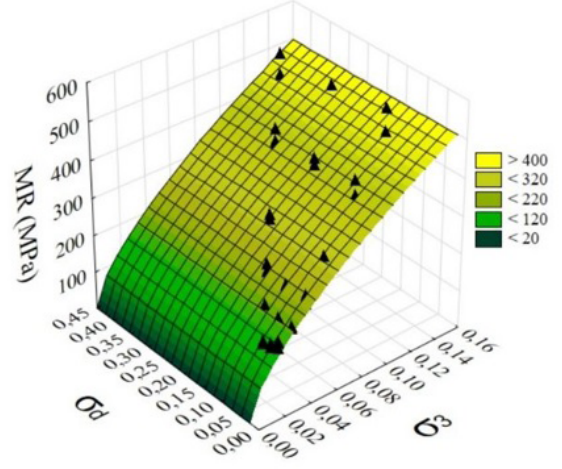

B

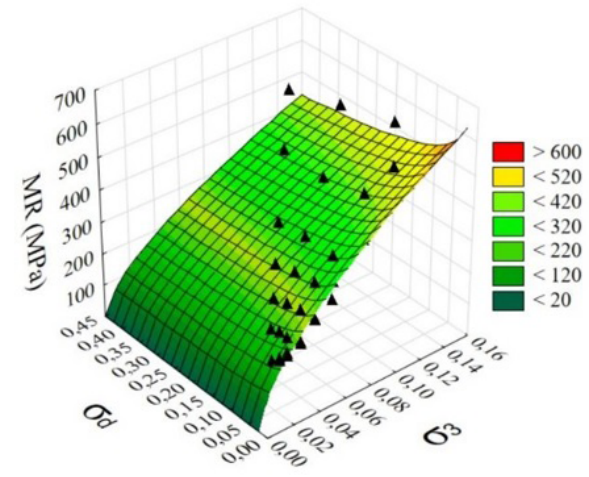

D

Figura 6 Gráficos Modelo Composto para A. Solo; B. SB 1; C. SB 2; D. SB 3. 


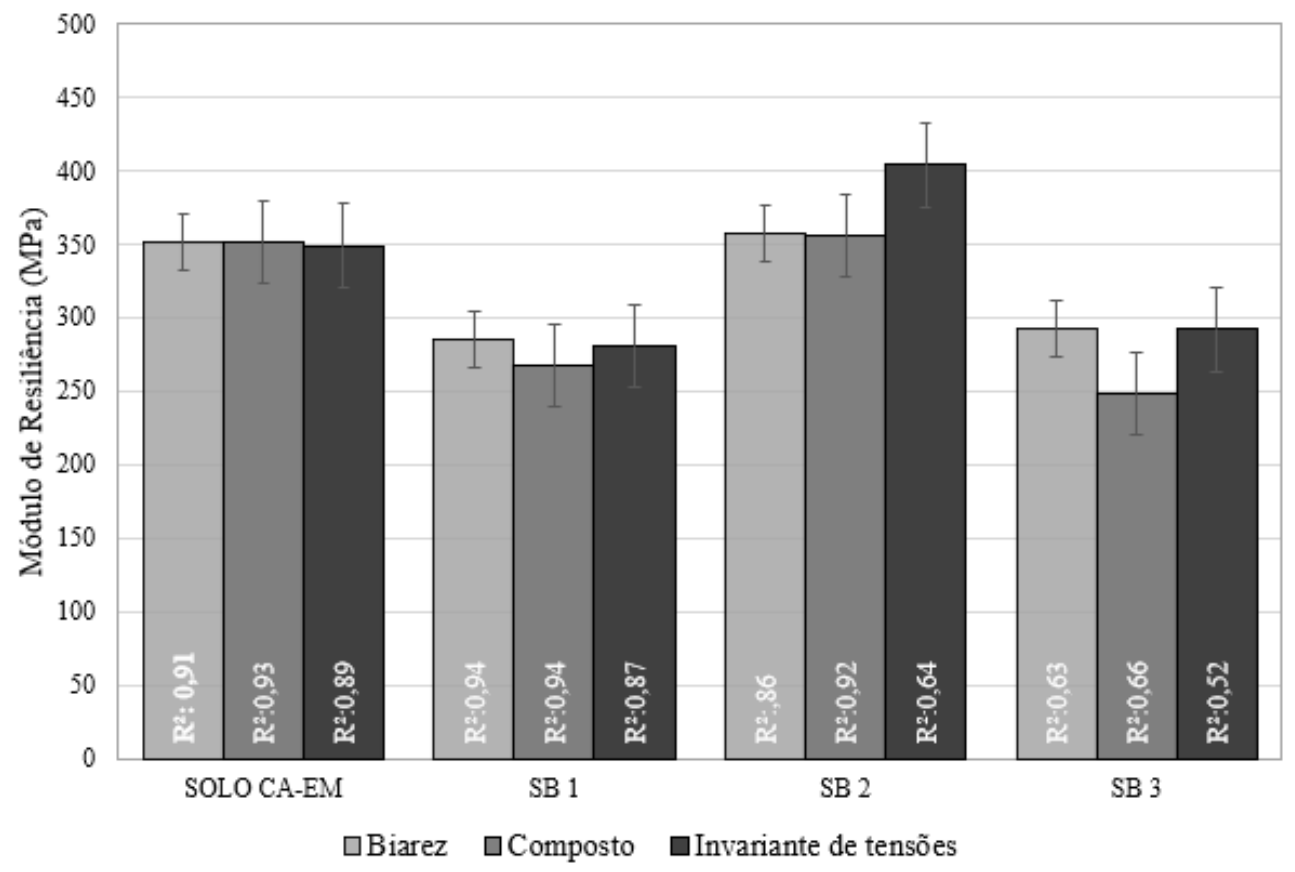

Figura 7 Valores médios de MR para cada um dos modelos estatísticos propostos.

solo e brita, indica a ocorrência de uma redução do módulo de resiliência com o aumento da tensão desvio, já o oposto ocorre quando vinculado ao solo natural, em que k3 é positivo. A mistura intermediária ainda teve resultados de MR médio de 357 MPa pelo modelo de Biarez e $404 \mathrm{MPa}$ para o Invariante de Tensões.

Ao avaliar o desempenho das misturas de acordo com modelo Composto, constata-se que mistura SB2 obteve melhor desempenho em comparação às demais misturas (SB 1: $267 \mathrm{MPa}$ e SB 3: $248 \mathrm{MPa}$ ), com um valor em torno de $25 \%$ maior a SB1 e $30 \%$ superior em relação a SB3. Isto posto, contata-se que a mistura com o melhor desempenho resiliente é a SB 2, composta por 30\% de solo e $70 \%$ de brita descontínua.

Os resultados comprovam que a definição de uma graduação "ótima" é um dos aspectos mais importantes da mistura. A composição de SB 2 indica uma situação em que os finos tendem a preencher os espaços vazios, permitindo ainda o contato entre os materiais britados, consequentemente, um bom comportamento resiliente. Nas amostras com presença de agregados em porcentagens mais elevadas, caso da mistura SB1, há um provável aumento do volume de vazios que não são preenchidos por solo, resultando em uma falta de coesão e redução do MR. Nas amostras da mistura com maior teor de solo, SB3, percebe-se também um decréscimo de seu desempenho resiliente, possivelmente pela diminuição do contato entre os agregados.

\subsection{Análise Mecanicista}

A mistura com o melhor desempenho resiliente (SB 2), foi selecionada para integrar a estrutura alternativa, com o método Composto, por possuir maior aplicabilidade no Brasil. Salienta-se que para compor o pavimento referência no software, foi necessário adaptar a camada de sub-base usada em campo para um material representativo (BG $\mathrm{C} 2$ ), tendo em vista a inexistência de ensaios triaxiais dinâmicos com amostras de macadame por limitações de dimensões dos equipamentos. A representação gráfica das seis estruturas para tráfegos baixo, médio e real, Figura 3, consideram subleito constituído pelo solo de Cruz Alta.

A fim de comprovar a melhora de comportamento em relação à fadiga, a Tabela 7 apresenta um resumo da análise comparativa da porcentagem de área trincada do pavimento real e do pavimento alternativo após os 10 anos de projeto.

Ao analisar os percentuais de área trincada que foram exibidos por cada estrutura no fim do período de projeto, verifica-se que o comportamento quanto à fadiga em pavimentos com um menor volume de tráfego aplicado é mais satisfatório, sendo que as estruturas concebidas com base e sub-base em BGS tiveram desempenhos inferiores neste ponto em relação às estruturas que são compostas pelas misturas SB. Em $5 \times 10^{6}$ solicitações, o percentual de área trincada do pavimento tradicional é de $6,6 \%$, enquanto o pavimento com apenas uma camada de base de SB 2 atinge $3,6 \%$. 
Com o aumento do tráfego há também um crescimento significativo na diferença de desempenho das estruturas. Verifica-se que, de acordo com o MeDiNa, o pavimento projetado originalmente tem o término de sua vida útil depois de 70 meses de funcionamento e, após dez anos, um total de $74,4 \%$ de área trincada. Em oposição, o pavimento com ausência de sub-base e substituição da base original por SB 2, teria condições aceitáveis em função da fadiga durante os seus dez anos de vida útil, com um total de $19,4 \%$ de área trincada. Neste contexto, com as estruturas de pavimentos submetidas a um nível tão elevado de tráfego, como o $\mathrm{N}$ de projeto, a E06 apresentaria um desempenho quanto à fadiga $73,92 \%$ melhor do que a E05.

Apesar do comprovado excelente desempenho resiliente das misturas solo-brita, a sua substituição em campo tem aspectos negativos relevantes. Além de uma execução mais complexa, reconhecidamente o comportamento dessas misturas na presença de umidade em excesso, resultam na perda de suas características, por supostamente possuírem maior dificuldade de alívio da poropressão e consequente deficiência de drenagem do pavimento. Portanto, simples trincas na camada asfáltica ou um projeto de drenagem mal realizado, poderiam acarretar na ruína do pavimento antes do esperado.

\subsection{Análises Econômicas}

Os levantamentos de custos realizados através de diretrizes do DAER e do SICRO, apresentados na Tabela 8, considera as estruturas tidas como referência sendo as que possuem em suas camadas de base e sub-base BGS (E1, E3 e E5) e as estruturas alternativas como as estruturas propostas a partir das misturas estudadas (E2, E4 e E6) às duas estruturas de pavimento são apresentados na Tabela 8. Considerando que o pavimento analisado faz parte da rede rodoviária estadual do Rio Grande do Sul, os dois métodos utilizados para a avaliação econômica do pavimento são cabíveis, por se tratarem de sistemas de uso regional (Levantamento de Custos do DAER, 2019) e nacional (SICRO outubro de 2019).

Com exceção da mudança nas camadas de base e sub-base, a estrutura dos pavimentos é igual em todas as demais porções. Por isso, através do levantamento de custos do DAER, buscou-se apenas avaliar a diferença de custos para execução das camadas supracitadas. Neste sentido, o pavimento alternativo resulta em uma proposta mais interessante, com um custo de execução de $1 \mathrm{~km}$ de $\mathrm{R} \$ 73.353,00$, enquanto o pavimento referência custaria R\$ $189.439,60$ para cada quilômetro. Esta diferença corresponde a 116 mil reais, uma redução de $61 \%$ do valor nas camadas analisadas.

Assim, a estrutura referência, demandaria um investimento de $\mathrm{R} \$ 1.299 .174,04$ para cada quilômetro construído, enquanto o pavimento que substitui as camadas de base e sub-base pela mistura SB2, custaria $\mathrm{R} \$ 1.120 .730,21$, ou seja, uma economia de $13,75 \%$ do custo total da obra. Estas diferenças são perfeitamente compreensíveis ao considerar a subtração de $19 \mathrm{~cm}$ de camada de sub-base composta por macadame seco.

\begin{tabular}{lccccc}
\hline Análise & Estrutura & $\begin{array}{c}\text { \% de área trincada } \\
\text { ao final de } \mathbf{1 0} \text { anos }\end{array}$ & $\begin{array}{c}\text { N } \\
\text { (solicitações) }\end{array}$ & $\begin{array}{c}\text { N limite } \\
\text { (solicitações) }\end{array}$ & $\begin{array}{c}\text { Tempo } \\
\text { (meses) }\end{array}$ \\
\hline E01 & Referência & 2,6 & $1,00 \times 10^{6}$ & $1,00 \times 10^{6}$ & 120 \\
E02 & Alternativa & 1,7 & & $1,00 \times 10^{6}$ & 120 \\
E03 & Referência & 6,6 & $5,00 \times 10^{6}$ & $5,00 \times 10^{6}$ & 120 \\
E04 & Alternativa & 3,6 & & $5,00 \times 10^{6}$ & 120 \\
E05 & Referência & 74,4 & $3,57 \times 10^{7}$ & $1,88 \times 10^{7}$ & 70 \\
E06 & Alternativa & 19,4 & $3,57 \times 10^{7}$ & 120 \\
\hline
\end{tabular}

Tabela 7 Parâmetros resilientes para o conjunto de amostras de acordo com os modelos.

\begin{tabular}{lccc}
\hline Estrutura & Sistema utilizado & Custo (R\$) & Observações \\
\hline Referência & DAER & $189.439,60$ & Somente os custos de aplicação da \\
Alternativa & & $73.353,00$ & base e sub-base das estruturas \\
Referência & SICRO - DNIT & 1.299 .17404 & Custos diretos da implantação da \\
Alternativa & & $1.120 .730,21$ & estrutura inteira \\
\hline
\end{tabular}

Tabela 8 Orçamentos através do DAER e SICRO. 


\section{Considerações Finais}

O pavimento deve ser dimensionado de forma que todas as suas camadas evidenciem um comportamento satisfatório, em relação ao carregamento em um dado período de projeto. Além disso, busca-se uma estrutura que apresente um investimento adequado em relação ao local de aplicação. Assim sendo, esta pesquisa analisou o comportamento resiliente do solo utilizado no subleito dos pavimentos e de três misturas de solo-brita, obtidos através do ensaio triaxial de cargas repetidas, dando ênfase à melhor mistura, adotando-a para diferentes níveis de tráfego, através do dimensionamento no software MeDiNa e análise econômica pelos métodos do DAER e SICRO, permitindo às seguintes conclusões:

I. O solo foi classificado respectivamente pela TRB/ AASHTO e SUCS como A-7-6 (11) e MH, materiais considerados de comportamento de sofrível a mau para emprego em pavimentos, enquanto, através da MCT, o mesmo material foi classificado como LG' (com grande potencial para emprego em pavimentos).

II. O desempenho resiliente médio do solo foi superior aos das misturas SB 1 e SB 3, comprovando excelente potencial para a aplicação em estruturas de pavimento. $\mathrm{O}$ modelo Composto, que possui influência de mais de uma variável de análise, apresentou melhores correspondentes em relação à mistura, representando melhores coeficientes de correlação em relação aos outros modelos avaliados neste trabalho. Além deste, o modelo de Biarez e Invariante de Tensões apresentaram bons coeficientes de correlação, ao passo que o modelo de Svenson apresentou $\mathrm{R}^{2}$ bem inferiores. Para o modelo Composto, de melhor correspondência, o solo e a misturas SB1, SB2 e SB3 obtiveram valores de MR médio de $351 \mathrm{Mpa}, 267 \mathrm{MPa}, 356 \mathrm{MPa}$ e 248 MPa na devida ordem.

III. A mistura SB 2, composta por 30\% de solo laterítico e $70 \%$ de brita descontínua, obteve o melhor desempenho frente aos carregamentos, com maiores módulos de resiliência em relação às demais amostras e foi considerada nas análises de comportamento dos pavimentos quanto ao trincamento à fadiga. Nessa análise, a estrutura alternativa mostrou resultados superiores para os diferentes tráfegos solicitados, ao ser comparado ao pavimento referência. Ainda que para tráfegos leves e médios, ambas as estruturas resultaram em percentuais de dano de área trincada dentro dos limites aceitáveis a sistemas arteriais principais, para o tráfego real, apenas a estrutura alternativa se mostrou eficiente.

IV. A concepção do pavimento alternativo apresentou maior economia pelas duas metodologias propostas para análise. Na implantação do pavimento como um todo (SICRO), a economia mostra-se menor em porcentagem, pois a parcela de custos da implantação das camadas de base e sub-base também são menores quando comparados às demais camadas do pavimento.

A aplicação de camadas de solo-brita em pavimentos, mostrou-se muito promissora nos aspectos relacionados ao comportamento resiliente, entretanto salienta-se que outros fatores importantes também devem ser observados. Ainda é necessária a compreensão do comportamento dos materiais quanto à deformação permanente $\mathrm{e}$, no que tange à execução, é fundamental o projeto de um sistema de drenagem eficiente e a atenção para a qualidade das demais camadas que irão compor o pavimento, pois a ação do efeito da água além do especificado pode levar o pavimento à ruína.

\section{Agradecimentos}

À Rede de tecnologia em asfaltos ANP/PETROBRAS e ao $\mathrm{MCTI} / \mathrm{CNPq}$ pelo apoio às pesquisas do Grupo de Estudos e Pesquisas em Pavimentação e Segurança Viária - GEPPASV da Universidade Federal de Santa Maria.

\section{Referências}

AASHTO. 2004. American Association of State Highway and Transportation Officials e Federal Highway Administration. NCHRP 1-37A: Guide for mechanistic-empirical design of new and rehabilitated pavement structures. Estados Unidos da América, 219 p.

ABNT. 2011. Associação Brasileira de Normas Técnicas. NBR 5564: Via férrea - Lastro ferroviário-Requisitos e métodos de ensaio. Rio de Janeiro, 26 p.

ABNT. 2016a. Associação Brasileira de Normas Técnicas. NBR 6459: Solos - Determinação do Limite de Liquidez. Rio de Janeiro, 5 p.

ABNT. 2016b. Associação Brasileira de Normas Técnicas. NBR 6508: Grãos de pedregulho retidos na peneira de abertura 4,8 mm - Determinação da massa específica, da massa específica aparente e da absorção de água. Rio de Janeiro, 8 p.

ABNT. 2016c. Associação Brasileira de Normas Técnicas. NBR 7180: Solos - Determinação do Limite de Plasticidade. Rio de Janeiro, 3 p.

ABNT. 2016d. Associação Brasileira de Normas Técnicas. NBR 7181: Solos - Análise Granulométrica. Rio de Janeiro, 12 p.

ANP. 2020. Agência Nacional do Petróleo, Gás Natural e Biocombustíveis. Preços e defesa da concorrência - Preços médios ponderados mensais (produto / região geográfica) 
(2008-2020). Disponível em: <http://www.anp.gov.br/precose-defesa-daconcorren cia/precos/precos-de-distribuicao $>$. Acesso em: 20 abr. 2020.

Back, A.H. 2018. Avaliação das propriedades químicas e geomecânicas de agregados oriundos de jazidas de rochas de diferentes litologias do estado do RS. Programa de Pósgraduação em Engenharia Civil, Universidade Federal de Santa Maria, Dissertação de Mestrado, 179 p.

Barros, L.M.; Lima, C.D.A.; Nascimento, L.A.H.; Motta, L.M.G. \& Aragão, F.T.S. 2019. Aspectos dos ensaios de deformação permanente e a perspectiva brasileira para utilização no dimensionamento de pavimentos asfálticos. In: ANAIS $33^{\circ}$ Congresso de Pesquisa e Ensino em Transporte da ANPET, Balneário Camboriú, ANPET, p. 1156-1167.

Bernucci, L.B.; Motta, L.M.G.; Ceratti, J.A.P. \& Soares, J.B. 2010. Pavimentação asfáltica: formação básica para engenheiros. Rio de Janeiro, ABEDA. 504 p.

Biarez, J. 1962. Contribution à l'etude des propriétés mécaniques des sols et des matériaux pulvérulents. Faculté des Sciences, l'Université de Grenoble, Thèse de Doctorat.

Ceratti, J.A.P.; Bernucci, L.B.; Soares, J.B. 2015. Utilização de ligantes asfálticos em serviços de pavimentação. Rio de Janeiro, ABEDA. 166 p.

DAER. 2001. Departamento Autônomo de Estradas de Rodagem. EL 108: Determinação do indice de lamelaridade. Rio Grande do Sul, $4 \mathrm{p}$.

DAER. 2019. Departamento Autônomo de Estradas de Rodagem. Custo unitário do serviço-Composição de serviços referencial de preços maio/2019 com desoneração. Rio Grande do Sul, $55 \mathrm{p}$.

DNER. 1996. Departamento Nacional de Estradas de Rodagem. CLA 259: Classificação de solos tropicais para finalidades rodoviárias utilizando corpos-de-prova compactados em equipamento miniatura. Rio de Janeiro, 6 p.

DNER. 1997a. Departamento Nacional de Estradas de Rodagem. ME 54: Equivalente de areia. Rio de Janeiro, 10 p.

DNER. 1997b. Departamento Nacional de Estradas de Rodagem. ME 195: Agregado-determinação da absorção e da massa específica de agregado graúdo. Rio de Janeiro, 6 p.

DNER. 1998a. Departamento Nacional de Estradas de Rodagem. ME 35: Agregados - determinação da abrasão "Los Angeles". Rio de Janeiro, $6 \mathrm{p}$.

DNER. 1998b. Departamento Nacional de Estradas de Rodagem. ME 83: Agregado-análise granulométrica. Rio de Janeiro, $5 \mathrm{p}$.

DNIT. 2018. Departamento Nacional de Infraestrutura de Transportes. ME 134: Pavimentação-Solos-Determinação do módulo de resiliência - Método de ensaio. Rio de Janeiro, $18 \mathrm{p}$.

DNIT. 2019. Departamento Nacional de Infraestrutura de Transportes. Custos e pagamentos - SICRO/Sul (outubro de 2019). Disponível em: <https://www.dnit.gov.br/custose-pagamentos/sicro/sul/rio-grande-do-sul/2019-1/outubro/ outubro-2019>. Acesso em: 20 abr. 2020.

Franco, F.A.C.P. \& Motta, L.M.G. 2018. MeDiNa-Método de Dimensionamento Nacional. Manual de utilização. Versão 1.0.0. Rio de Janeiro. 70 p.
Esfahani, M.A. 2018. Evaluating the feasibility, usability, and strength of recycled construction and demolition waste in base and subbase courses. Road Materials and Pavement Design, 21(1): 156-178.

Guimarães, A.C.R; Motta, L.M.G. \& Medina, J. 2001. Estudo de deformação permanente em solo típico de subleito de rodovia brasileira. In: $33^{\text {a }}$ REUNIÃO ANUAL DE PAVIMENTAÇÃO. Florianópolis, ABPv, p. 336-354.

Li, D. \& Selig, E. 1994. Resilient modulus for fine-grained subgrade soils. Journal of geotechnical engineering, 120(6): 939-957.

Lima, C.D.A.; Motta, L.M.G.; Guimarães, A.C.R. \& Aragão, F.T.S. 2018. Contribution to the study of Brazilian tropical soils as pavement materials. In: ANAIS $13^{\text {a }}$ CONFERENCE ON ASPHALT PAVEMENTS, Fortaleza, ISAP, p. 1-8.

Lima, C.D.A.; Motta, L.M.G. \& Aragão, F.T.S. 2019. Effects of Compaction Moisture Content on Permanent Deformation of Soils Subjected to Repeated Triaxial Load Tests. Transportation Research Record, 2673(2): 466-476.

Medina, J. \& Preussler, E.S. 1980. Características resilientes de solos em estudos de pavimentos. Solos e Rochas, 3(1): 3-26.

Medina, J. \& Motta, L.M.G. 2015. Mecânica dos Pavimentos. Rio de Janeiro, Interciência, 638 p.

Motta, L.M.G. 1991. Método de dimensionamento de pavimentos flexíveis: critério de confiabilidade e ensaios de cargas repetidas. Programa de Pós-graduação em Engenharia Civil, Universidade Federal do Rio de Janeiro, Tese de Doutorado, $336 \mathrm{p}$.

Norback, C. \& Motta, L.M.G. 2018. Caracterização do módulo de resiliência e da deformação permanente de duas misturas solo-brita. In: ANAIS $32^{\circ}$ Congresso de Pesquisa e Ensino em Transporte da ANPET, 2018. Gramado, ANPET, p. 20982109.

Petrobras. 2010. Rede temática de asfalto. Manual de execução de trechos monitorados. [S.1.]: 2010.

Pezo, R.F.; Claros, G.; Hudson, W.R. \& Stoke, I.K.H. 1992. Development of reliable resilient modulus test for subgrade and non-granular subbase materials for use in routine pavement design. Research Report, 1177(1): 190-194.

Prado, K.; Albuquerque, F.S. \& Almeida, G.B.O. 2020. Influence of granulometry, cement content, and moisture in the mechanical performance of the cemented crushed stone. Transportes, 28(1): 81-98.

Rossato, F.P. 2015. Avaliação do fenômeno de fadiga e das propriedades elásticas de misturas asfálticas com diferentes ligantes em variadas temperaturas. Programa de Pósgraduação em Engenharia Civil, Universidade Federal de Santa Maria, Dissertação de Mestrado, 221 p.

Seed, H.B.; Mitry, F.G.; Monismith, C.L. \& Chan, C.K. 1967. Prediction of flexible pavement deflections from laboratory repeated load tests. National Cooperative Highway Research Program, 35(1): 112.

Soares, J.B.; Moreno, A.M. \& Motta, L.M.G. 2009. Aspectos gerais de métodos de dimensionamento de pavimentos asfálticos de vários países e a relação com um novo método brasileiro. Revista Pavimentação, 4(1): 20-35. 
Svenson, M. 1980. Ensaios triaxiais dinâmicos de solos argilosos. Programa de Pós-Graduação em Engenharia Civil, Universidade Federal do Rio de Janeiro, Dissertação de Mestrado, $135 \mathrm{p}$.

Vertematti, E. 1988. Contribuição ao conhecimento geotécnico de solos da Amazônia com base na investigação de aeroportos e metodologia MCT e resiliente. Programa de Pós-Graduação em Ciência, Instituto Tecnológico da Aeronáutica, Tese de Doutorado, $276 \mathrm{p}$.
Villibor, D.F.; Nogami, J.S.; Cincerre, J.R.; Serra, P.R.M. \& Neto, A.Z. 2009. Pavimentos de Baixo Custo para Vias Urbanas. São Paulo, Arte \& Ciência, 196 p.

Werk, S.M.S. 2000. Estudo da influência dos métodos de compactação no comportamento resiliente de solos. Programa de Pós-Graduação em Engenharia Civil, Universidade Federal do Rio Grande do Sul, Dissertação de Mestrado, 118 p.

Yoder, E.J. \& Witczak, M.W. 1975. Principles of pavement design. New York, John Wiley \& Sons. 711 p.

\section{Como citar:}

Mello, L.B.; Chiarello, G.P.; Pascoal, P.T.; Baroni, M. \& Specht, L.P. 2021. Solo-Brita em Bases de Pavimentos Flexíveis: Avaliação quando à Fadiga Utilizando o MeDiNa. Anuário do Instituto de Geociências, 44: 35192. DOI 1982-3908_2021_44_35192 